\title{
SCHUR'S THEOREMS ON COMMUTATIVE MATRICES
}

\author{
N. JACOBSON
}

In $1905 \mathrm{I}$. Schur ${ }^{1}$ proved that the maximum number $N(n)$ of linearly independent commutative matrices of $n$ rows and columns is given by the formula $N(n)=\left[n^{2} / 4\right]+1=\nu^{2}+1$ if $n=2 \nu$ and $=\nu(\nu-1)+1$ if $n=2 \nu-1$. Schur also determined the sets of linearly independent commutative matrices containing $N(n)$ elements. In this note we give a simpler derivation of Schur's results and an extension of these results from algebraically closed fields to arbitrary fields.

If $A_{1}, \cdots, A_{N(n)}$ is a set of linearly independent commutative matrices, the set $\mathfrak{A}$ of matrices $\sum A_{i} \phi_{i}$ where $\phi_{i}$ is arbitrary in the underlying field $\Phi$ is a commutative subalgebra containing the identity of the matrix algebra $\Phi_{n}$. Hence $N(n)$ is the maximal dimensionality of commutative subalgebras of $\Phi_{n}$. It is easy to see that $N(n) \geqq\left[n^{2} / 4\right]$ +1 . For consider the set $Z_{n}$ of matrices

$$
\left(\begin{array}{ll}
0 & A \\
0 & 0
\end{array}\right)
$$

where if $n=2 \nu, A$ is arbitrary in $\Phi_{\nu}$ and if $n=2 \nu-1, A$ is an arbitrary matrix of $\nu$ rows and $\nu-1$ columns. Thus $\operatorname{dim} B_{n}=\left[n^{2} / 4\right]$. It may be verified that $\mathbb{Z}_{n}$ is a zero algebra. Hence the algebra $\mathfrak{B}_{n}$ obtained by adjoining 1 to $Z_{n}$ is a commutative algebra of dimensionality $\left[n^{2} / 4\right]+1$. We remark also that if $n=2 \nu-1$ we may replace $3_{n}$ by the algebra $\bar{B}_{n}$ of matrices of the form (1) in which $A$ is an arbitrary matrix of $\nu-1$ rows and $\nu$ columns. We denote by $\overline{\mathfrak{B}}_{n}$ the extension of $\overline{3}_{n}$ obtained by adjoining 1 .

To prove that $N(n) \leqq\left[n^{2} / 4\right]+1$ it suffices to assume that $\Phi$ is algebraically closed. For if $A_{1}, \cdots, A_{N(n)}$ are linearly independent and commutative in $\Phi_{n}$, then they have these properties in $\Sigma_{n}$ for any extension field $\Sigma$ of the field $\Phi$. Thus $N(n, \Phi) \leqq N(n, \Sigma)$. We shall therefore assume that $\Phi$ is algebraically closed. Let $\mathfrak{A}$ be a commutative subalgebra of $\Phi_{n}$ containing the identity and let $N$ be the dimensionality of $\mathfrak{A}$ over $\Phi$. We suppose first that $\mathfrak{A}$ is an indecomposable algebra of matrices. Then it is known that by replacing $\mathfrak{A}$ by a similar set we may suppose that the matrices of $\mathfrak{A}$ have the form

Received by the editors January 14, 1944.

${ }^{1}$ Zur Theorie vertauschbaren Matrizen, J. Reine Angew. Math. vol. 130 (1905) pp. 66-76. 


$$
\left(\begin{array}{ccc}
{ }^{\alpha} & & \\
& \alpha & \\
& & \cdot \\
0 & & \alpha
\end{array}\right) .
$$

Thus $\mathfrak{A}=(1)+\mathfrak{N}$ where $\mathfrak{N}$ is a nilpotent algebra of matrices in proper triangular form, that is, of the form (2) in which $\alpha=0$. Evidently $\operatorname{dim} \mathfrak{N}=N-1$.

Let the $k_{1}$ th column $\left(k_{1}>1\right)$ be the first column for which there exists a matrix $U_{1 k_{1}}$ in $\Re$ with element in the $\left(1, k_{1}\right)$ position not equal to 0 . We may suppose that the element in the $\left(1, k_{1}\right)$ position of $U_{1 k_{1}}$ is 1 . We normalize $U_{1 k_{1}}$ further by using the following lemma.

LEMMA 1. Let $U \in \Phi_{n}$ and let $V$ be the matrix obtained from $U$ by adding the kth column multiplied by $\theta$ to the lth column $(k \neq l)$ and then subtracting the lth row multiplied by $\theta$ from the kth row. Then $U$ and $V$ are similar.

We have $V=S^{-1} U S$ where $S=1+e_{k l} \theta, e_{k l}$ the matrix with 1 in the $(k, l)$ position and 0 's elsewhere.

We may apply this lemma to $U_{1 k_{1}}$ and replace it by a matrix whose first row is $e_{k_{1}}=(0, \cdots, 1,0, \cdots, 0)$ where the 1 is in the $k_{1}$ th column. The operations required for this purpose are additions of multiples of the $k_{1}$ th column to later columns and additions to the $k_{1}$ th row of later rows. These operations replace $\mathfrak{N}$ by a properly triangular set of matrices $\mathfrak{N}^{\prime}$ similar to $\mathfrak{N}$ such that all the elements in the $(1, j)$ position with $j<k_{1}$ in $\mathfrak{R}^{\prime}$ are 0 and such that $\mathfrak{N}^{\prime}$ contains a matrix $V_{1 k_{1}}\left(\right.$ similar to $U_{1 k_{1}}$ ) whose first row is $e_{k_{1}}$. Now let $\mathfrak{P}^{\prime}$ be the subspace of $\mathfrak{N}^{\prime}$ of matrices in which the elements in the $\left(1, k_{1}\right)$ position are 0 and suppose that the $k_{2}$ th column $\left(k_{2}>k_{1}\right)$ is the first column for which there is a matrix $U_{1 k_{2}}$ in $\mathfrak{P}^{\prime}$ with element in the $\left(1, k_{2}\right)$ place not equal to 0 . Evidently any matrix in $\mathfrak{N}^{\prime}$ has the form $V_{1 k_{1}} \beta_{1}+P^{\prime}, P^{\prime}$ in $\mathfrak{B}^{\prime}$. We now apply to $U_{1 k_{2}}$ the process used before for $U_{1 k_{1}}$ and replace it by a matrix $V_{1 k_{2}}$ similar to it and having $e_{k_{2}}$ for first row. The set $\mathfrak{N}^{\prime}$ will be transformed into a set $\mathfrak{N}^{\prime \prime}$ of properly triangular matrices and $V_{1 k_{1}}$ changed into a new matrix which we shall again denote as $V_{1 k_{1}}$ with first row $e_{k_{1}}$. Any matrix in $\mathfrak{N}^{\prime \prime}$ has the form $A=V_{1 k_{1}} \beta_{1}+P^{\prime \prime}, P^{\prime \prime}$ in $\mathfrak{P}^{\prime \prime}$, the transform of the set $\mathfrak{P}^{\prime}$. It is clear that the elements in the $(1, j)$ position, $j<k_{2}$, for any matrix in $\mathfrak{B}^{\prime \prime}$ are 0 . Hence $A=V_{1 k_{1}} \beta_{1}+V_{1 k_{2}} \beta_{2}+S^{\prime \prime}$ where $S^{\prime \prime}$ is in the subspace $\widetilde{S}^{\prime \prime}$ of $\mathfrak{N}^{\prime \prime}$ of matrices having 0 in the $(1, j)$ position with $j \leqq k_{2}$. This process may be continued and proves the following lemma.

LEMMA 2. The set $\mathfrak{N}$ is similar to a set $\mathfrak{R}^{(r)}$ of properly triangular 
matrices that contain matrices $V_{1 k_{1}}, \cdots, V_{1 k_{r}}$ such that the first row of $V_{1 k_{i}}$ is $e_{k_{i}}, 1<k_{1}<k_{2}<\cdots<k_{r}$, and such that any matrix in $\mathfrak{N}^{(r)}$ has the form $\sum V_{1 k_{i}} \beta_{i}+Z$, where $Z$ has first row 0 .

Now let $\mathfrak{N}_{2}$ be the subset of $\mathfrak{N}^{(r)}$ of matrices $Z$ having first row 0 . Evidently $\mathfrak{R}^{(r)}=\left\{V_{1 k_{1}}, \cdots, V_{1 k_{r}}\right\}+\mathfrak{N}_{2}$ and the $V_{1 k_{i}}$ are linearly independent. Hence $\operatorname{dim} \mathfrak{N}^{(r)}=N-1=r+\operatorname{dim} \mathfrak{N}_{2}$. Now we note that if $Z \in \mathfrak{N}_{2}$, the first row of $V_{1 k_{i}} Z$ is the $k_{i}$ th row of $Z$ and the first row of $Z V_{1 k_{i}}$ is 0 . Hence the $k_{i}$ th row of every matrix $Z$ in $\mathfrak{N}_{2}$ is 0 .

We now repeat the argument for $\mathfrak{N}_{2}$. Then $\mathfrak{N}_{2}$ may be replaced by a set $\mathfrak{N}_{2}^{(s)}$ similar to $\mathfrak{N}_{2}$ such that (1) $\mathfrak{N}_{2}^{(s)}$ is properly triangular, (2) $\mathfrak{N}_{2}^{(s)}$ contains matrices $V_{2 l_{1}}, \cdots, V_{2 l}$, having first row 0 and second row $e_{l_{1}}, \cdots, e_{l_{2}}$, respectively, such that any matrix in $\mathfrak{N}_{2}^{(s)}$ has the form $\sum V_{2 l_{i}} \beta_{i}+Z$ where $Z$ is a matrix with first two rows 0 . Let $\mathfrak{N}_{3}$ denote the set of matrices $Z$. We assert that if $s=l_{i}$ or $s=k_{j}$ then the $s$ th row of $\mathfrak{N}_{3}$ is 0 . This is clear if $s=l_{i}$. Hence suppose that $s=k_{j} \neq$ any $l_{i}$. Then the matrices of $\mathfrak{R}_{2}$ all have $k_{j}$ th row 0 and the operations performed in passing from $\mathfrak{N}_{2}$ to $\mathfrak{N}_{2}^{(s)}$ do not affect this row. Hence the $k_{j}$ th row of every matrix in $\mathfrak{N}_{2}^{(s)}$ is 0 . Evidently $N-1=r+s+\operatorname{dim} \mathfrak{N}_{3}$.

We now write $k_{i}=k_{1 i}, l_{i}=k_{2 i}, r=r_{1}, s=r_{2}$. Then if we continue this process we see that $N-1$ is equal to the number of matrices in the following set

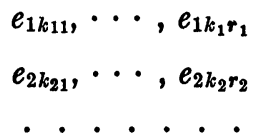

where $1<k_{11}<\cdots<k_{1 r_{1}}, 2<k_{21}<k_{22}<\cdots<k_{2 r_{2}}, \cdots$, and $r_{i}=0$ if $i=k_{j l}$ with $j<i$. Let $s_{1}, s_{2}, \cdots, s_{m}$ be the complete set of integers $k_{i j}$ arranged in increasing order. Then it is clear that $N-1$ $\leqq N\left(s_{1}, s_{2}, \cdots, s_{m}\right)$, the number of matrices in the set

$$
\begin{aligned}
& e_{1 s 1}, e_{2 s 1}, \cdots, e_{s 1-1, s_{1}} \\
& e_{1 s_{2}}, e_{2 s_{2}}, \cdots, e_{s 1-1, s_{2}}, e_{s 1+1, s_{2}}, \cdots, e_{s_{2}-1, s_{2}}
\end{aligned}
$$

Evidently

$$
\begin{aligned}
N\left(s_{1}, s_{2}, \cdots, s_{m}\right) & =\left(s_{1}-1\right)+\left(s_{2}-2\right)+\cdots+\left(s_{m}-m\right) \\
& =\sum s_{i}-m(m+1) / 2 .
\end{aligned}
$$

Hence we have 


$$
\begin{aligned}
N-1 & \leqq N\left(s_{1}, \cdots, s_{m}\right) \leqq N(n-m+1, \cdots, n) \\
& =m(n-m) .
\end{aligned}
$$

Now $m(n-m)$ attains its maximum value for $m=[n / 2]$. If $n=2 \nu$ this maximum is $\nu^{2}$ and if $n=2 \nu-1$, it is $\nu(\nu-1)$. Thus the maximum value is $\left[n^{2} / 4\right]$. This proves for indecomposable algebras $\mathfrak{A}$ the following theorem.

THEOREM 1. If $\mathfrak{A}$ is a commutative subalgebra of $\Phi_{n}, \operatorname{dim} \mathfrak{A} \leqq\left[n^{2} / 4\right]$ +1 .

If $\mathfrak{A}$ is decomposable we suppose that the matrices of $\mathfrak{A}$ have the form

$$
\left(\begin{array}{ll}
A & 0 \\
0 & B
\end{array}\right)
$$

where $A \in \Phi_{n_{1}}$ and $B \in \Phi_{n_{2}}, n_{i} \geqq 1, n_{1}+n_{2}=n$. We may assume that the theorem holds for the $\Phi_{n_{i}}$.

Case 1. $n=2 \nu-1, \quad n_{1}=2 \nu_{1}-1, \quad n_{2}=2 \nu_{2}$. Here $\nu=\nu_{1}+\nu_{2}$ and $N \leqq \nu_{1}\left(\nu_{1}-1\right)+1+\nu_{2}^{2}+1 \leqq \nu(\nu-1)+1$. Equality holds between the last two terms only when $n=3$.

Case 2. $n=2 \nu, n_{1}=2 \nu_{1}-1, n_{2}=2 \nu_{2}-1$. Here $\nu=\nu_{1}+\nu_{2}-1$ and $N \leqq \nu_{1}\left(\nu_{1}-1\right)+1+\nu_{2}\left(\nu_{2}-1\right)+1 \leqq \nu^{2}+1$. Equality holds only if $n=2$.

Case 3. $n=2 \nu, n_{1}=2 \nu_{1}, n_{2}=2 \nu_{2}$. Here $\nu=\nu_{1}+\nu_{2}$ and $N=\nu_{1}^{2}+1+\nu_{2}^{2}$ $+1<\nu^{2}+1$. Thus the theorem is proved.

We have also proved the following theorem.

THEOREM 2. The maximum number $N(n)$ of linearly independent commutative matrices of $n$ rows and columns is given by the formula $N(n)=\left[n^{2} / 4\right]+1$.

We shall investigate next the form of commutative subalgebras $\mathfrak{A}$ of $\Phi_{n}$ of the maximum dimensionality $N(n)$. Suppose first that $\mathfrak{A}$ has the structure $\mathfrak{A}=(1)+\mathfrak{N}$ where $\mathfrak{N}$ is a nilpotent algebra. Then it is known that by replacing $\mathfrak{A}$ by a similar set we may suppose that the matrices of $\mathfrak{R}$ are properly triangular. We may apply the above considerations to $\mathfrak{R}$. By (3), (4), (5) and (6) we see that if $n=2 \nu$ we must have $k_{11}=k_{21}=\cdots=k_{\nu 1}=\nu+1, \cdots, k_{1 \nu}=k_{2 \nu}=\cdots=k_{\nu \nu}=n$ as the set of $k$ 's in (3). If $n=2 \nu-1$ the set of $k$ 's is either $k_{11}=\cdots=k_{\nu 1}$ $=\nu+1, \cdots, k_{1 \nu-1}=\cdots=k_{\nu \nu-1}=n$ or $k_{11}=\cdots=k_{\nu-11}=\nu, \cdots$, $k_{1 \nu}=\cdots=k_{\nu-1 \nu}=n$. Suppose first that $n$ is even. Let $\mathfrak{N}^{(r)}(r=\nu)$ and $\mathfrak{N}_{2}$ be determined as before. It is clear that $\mathfrak{N}^{(r)}$ is similar to $\mathfrak{N}$ by a matrix in $\Phi_{n}$ and we need not assume here that $\Phi$ is algebraically closed. The matrices of $\mathfrak{N}_{2}$ have the form 


$$
\left.B=\left(\begin{array}{c|c}
0 \cdots 0 & \overbrace{0 \cdots 0}^{\nu} \\
R & \frac{A}{0}
\end{array}\right)\right\} \nu .
$$

Since $k_{21}=\nu+1$ it is clear that the second row of $R$ is 0 . Moreover the operations used to pass from $\mathfrak{N}_{2}$ to $\mathfrak{N}_{3}$ affect only the last $\nu$ rows and last $\nu$ columns of $\mathfrak{N}_{2}$. Hence the third row of $R$ is the same as the third row of the corresponding matrix in $\mathfrak{N}_{3}$. Since $k_{31}=\nu+1$ the third row of $R$ is 0 . Similarly the other rows of $R$ are 0 , and $R=0$ in (7). Now $\operatorname{dim} \mathfrak{N}_{2}=\nu^{2}-\nu$. Hence $\mathfrak{N}_{2}$ consists of all matrices of the form (7) in which $R=0$ and $A$ is arbitrary. Let

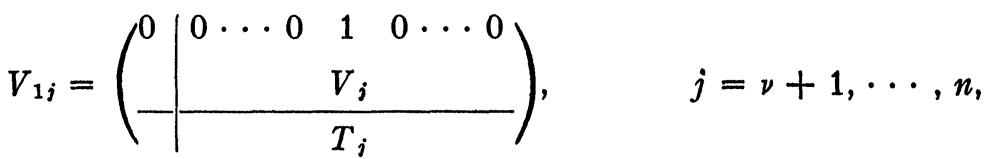

where the 1 is in the $j$ th column and $T_{j}$ is a properly triangular matrix. Since $V_{1 j} B=B V_{1 j}$ the following holds in $\Phi_{\nu}$ :

$$
\left(\begin{array}{c}
0 \cdots 0 \\
A
\end{array}\right) T_{j}=0
$$

Since $A$ is arbitrary, $T_{j}=0$. Thus $\mathfrak{N}^{(r)}$ is the set $\mathfrak{Z}_{n}$ and $\mathfrak{A}$ is similar to the algebra $\mathfrak{B}_{n}$ defined before. If $n$ is odd a similar argument shows that $\mathfrak{A}$ is similar either to $\mathfrak{B}_{n}$ or to $\overline{\mathfrak{B}}_{n}$.

We suppose now that $\mathfrak{A}$ is arbitrary. Evidently $\mathfrak{A}$ contains the identity matrix. Since $n>3$ by the proof of Theorem $1, \mathfrak{A}$ is indecomposable. Moreover if $\Omega$ is the algebraic closure of $\Phi$ then $\mathfrak{A}_{\Omega}$ is an indecomposable algebra containing the identity. It follows that $\mathfrak{A}_{\Omega}$ is similar to a set of matrices of the form (1). Hence $\mathfrak{A}_{\Omega}=(1)+B$ where $\mathfrak{Z}$ is nilpotent and so $\mathfrak{A}_{\Omega}$ is similar to either $\mathfrak{B}_{n}(\Omega)$ or $\overline{\mathfrak{B}}_{n}(\Omega)$. Thus $\mathbb{B}$ is a zero algebra. Now let $\mathfrak{N}$ be the radical of the algebra $\mathfrak{A}$ and consider the semi-simple alg ebra $\overline{\mathfrak{P}}=\mathfrak{A}-\mathfrak{N}$. The extension $\overline{\mathfrak{A}}_{\Omega}$ is a homomorphic image of $\mathfrak{A}_{\Omega}$. Hence $\overline{\mathfrak{A}}_{\Omega}=(1)+\overline{\overline{3}}$ where $\overline{\overline{3}}$ is a zero algebra. The structure of $\overline{\mathfrak{A}}$ is given by the following lemma.

LEMMA 3. If $\overline{\mathfrak{X}}$ is a semi-simple commutative algebra such that $\overline{\mathfrak{A}}_{\Omega}=(1)+\overline{\mathrm{X}}$ where $\overline{\bar{B}}$ is a zero algebra, then either $\overline{\mathfrak{Y}}=(1)$ or $\Phi$ is an imperfect field of characteristic 2 and $\overline{\mathfrak{U}}=\Phi(x)$ where $x^{2}=\xi$, a nonsquare in $\Phi$.

Since $\mathfrak{A}$ is semi-simple, $\overline{\mathfrak{X}}$ is a direct sum of fields, but since $\overline{\mathfrak{A}}_{\Omega}$ has only one idempotent element, $\overline{\mathfrak{A}}$ is a field. Let $\overline{\mathfrak{X}}>(1)$. Then $\overline{\mathfrak{X}}$ has no 
separable subfields, for if $\Sigma$ were such a subfield $\Sigma_{\Omega}$ is a direct sum of fields and $\overline{\mathfrak{P}}_{\Omega}$ would contain more than one idempotent element. Thus $\Phi$ has characteristic $p \neq 0$ and $\overline{\mathfrak{A}}$ contains an element $x$ such that $x^{p}=\xi$ is in $\Phi$ where $\xi$ is not a $p$ th power in $\Phi$. Now there exists an element $\eta$ in $\Omega$ such that $\eta^{p}=\xi$ and hence the element $z=x-\eta$ in $\overline{\mathfrak{A}}_{\Omega}$ is nilpotent of index $p$. Since $\bar{B}$ is a zero algebra, $p=2$. It follows readily that in this case $\mathfrak{A}=\Phi(x), x^{2}=\xi$.

This lemma shows that unless $\Phi$ is an imperfect field of characteristic 2 any commutative subalgebra $\mathfrak{A}$ of $\Phi_{n}(n>3)$ of maximum dimensionality has a difference algebra with respect to its radical $\mathfrak{N}$ of dimensionality 1 . Since $\mathfrak{A}$ contains the identity, $\mathfrak{A}=(1)+\mathfrak{N}$. As we have seen, this implies that $\mathfrak{A}$ is similar to either $\mathfrak{B}_{n}$ or to $\overline{\mathfrak{B}}_{n}$.

TheOREM 3. Suppose that $\Phi$ is not an imperfect field of characteristic 2 and let $n>3$. Then if $\mathfrak{A}$ is a subalgebra of $\Phi_{n}$ of maximum dimensionality $N(n), \mathfrak{A}$ is similar to $\mathfrak{B}_{n}$ if $n=2 \nu$ and $\mathfrak{A}$ is similar to either $\mathfrak{B}_{n}$ or $\overline{\mathfrak{B}}_{n}$ if $n=2 \nu-1 .^{2}$

As a consequence we have the following theorem.

Theorem 4. Let $\Phi, n$ and $\mathfrak{A}$ be as in Theorem 3. Then $\mathfrak{A}=(1)+\mathfrak{N}$ where $\mathfrak{N}$ is a zero algebra.

We remark finally that if $n$ is odd the sets $\mathfrak{B}_{n}$ and $\overline{\mathfrak{B}}_{n}$ are not similar. This may be seen by considering the sets $B_{n}$ and $\overline{\mathbb{B}}_{n}$. Let $\mathfrak{S}(\overline{\mathfrak{S}})$ be the space determined by the columns of the matrices of $\mathbb{B}_{n}\left(\bar{\Omega}_{n}\right)$. Then $\operatorname{dim} \mathfrak{S}=\nu$ and $\operatorname{dim} \overline{\mathfrak{S}}=\nu-1$. On the other hand if $3_{n}$ were similar to $\overline{\mathbf{B}}_{n}$ we would have $\operatorname{dim} \mathfrak{S}=\operatorname{dim} \overline{\mathfrak{S}}$. It follows that $\boldsymbol{B}_{n}$ and $\overline{\mathbf{B}}_{n}$ are not similar and hence $\mathfrak{B}_{n}$ and $\overline{\mathfrak{B}}_{n}$ are not similar. Thus in this case there are for $n=2 \nu-1>3$ two distinct classes in the sense of similarity of commutative subalgebras of dimensionality $N(n)$.

JoHNS HopkINS UNIVERsITY

2 If $n=2,3, \mathfrak{A}$ may be decomposable. The determination of these algebras is readily obtained. 